\title{
NOISE-DRIVEN SYNCHRONIZATION IN COUPLED MAP LATTICES
}

\author{
L. BARONI ${ }^{(1),(2)}$, R. $\operatorname{LIVI}^{(1),(2)}$, AND A. TORCINI ${ }^{(2),(3)}$ \\ ${ }^{1}$ Dipartimento di Fisica, Universitá di Firenze, L.go E. Fermi, 5 - I-50125 \\ Firenze, Italy \\ ${ }^{2}$ Istituto Nazionale di Fisica della Materia, UdR Firenze, L.go E. Fermi, 3 - \\ I-50125 Firenze, Italy \\ 3 Dipartimento di Energetica, Universitá di Firenze via S. Marta, 3 - I-50139 \\ Firenze, Italy
}

\begin{abstract}
Synchronization is shown to occur in spatially extended systems under the effect of additive spatio-temporal noise. In analogy to low dimensional systems, synchronized states are observable only if the maximum Lyapunov exponent $\Lambda$ is negative. However, a sufficiently high noise level can lead, in map with finite domain of definition, to nonlinear propagation of information, even in non chaotic systems. In this latter case the transition to synchronization is ruled by a new ingredient : the propagation velocity of information $V_{F}$. As a general statement, we can affirm that if $V_{F}$ is finite the time needed to achieve a synchronized trajectory grows exponentially with the system size $L$, while it increases logarithmically with $L$ when, for sufficiently large noise amplitude, $V_{F}=0$.
\end{abstract}

\section{Introduction}

Noise-driven phenomena like diffusion and growth processes, front propagation and interface dynamics are quite common and extensively studied problems in equilibrium and non-equilibrium statistical mechanics 1 . Usually, they are described in terms of stochastic PDE's, or probabilistic automata, where some random process is introduced as a model of white thermal noise 2.

More recently, noise has been shown to be able also to control chaotic fluctuations in low-dimensional dynamical systems, yielding synchronization of replicae driven by the same stochastic process. This problem has been widely investigated 0.9 . or the Lorenz system, coupled through the same additive noise. For sufficiently large noise amplitude, the invariant measure of both maps can be modified in such a way to favour the stabilization on the same stochastic orbit. Far from misterious, this effect can be explained and quantitatively predicted by observing that, for some noise amplitude, the Lyapunov exponent associated to the dynamics becomes negative, as brillantly argued by Pilovsky 0 . Clearly, this can happen only if the map dynamics has expanding and contracting regions, so that noise may amplify the role of the latter against the former 
by properly modifying the invariant measure of each map synchronization effect is obviously absent in uniformly hyperbolic maps, e.g. the Bernoulli shift.

In this paper we aim to extend this analysis to spatially extended dynamical systems under the influence of spatio-temporal noise. In particular, we have focused our attention on some specific coupled map lattice (CML) models 6 , that, in the absence of noise, exhibit dynamical phases characterized by robust statistical properties.

The main issues contained in this manuscript are hereafter summarized:

- A necessary condition for obtaining synchronization in CML models is that the maximum Lyapunov exponent $\Lambda$ becomes negative: this extends the validity of Pikovsky's criterion to spatially extended systems $\mathrm{Z}$.

- Additive spatio-temporal noise introduces effective discontinuities incoupled interval maps, that, for $\Lambda<0$, yield "stable-chaotic" phases 10 . In this case synchronization is ruled by a further indicator, the propagation velocity of information $V_{F} 11$.

\section{Models and Tools}

The general model of CML driven by the same realization of spatio-temporal noise is defined by the the following two-step evolution rule:

$$
\begin{aligned}
\tilde{x}_{i}^{t} & =(1-\varepsilon) x_{i}^{t}+\frac{\varepsilon}{2}\left(x_{i-1}^{t}+x_{i+1}^{t}\right) \\
x_{i}^{t+1} & =f\left(\tilde{x}_{i}^{t}\right)+\sigma \cdot \eta_{i}^{t}
\end{aligned}
$$

The real state variable $x_{i}^{t}$ depends on the discrete space and time indices $i=1,2, \cdots, L$ and $t=1,2, \cdots, T$, respectively. The diffusive coupling $\varepsilon$ varies in the interval $[0,1], \sigma$ is the amplitude of the normalized space-time dependent random variable $\eta_{i}^{t}$, uniformly distributed in the interval [-1,1], and $f(x)$ is a mapping from $\mathcal{S} \in \mathrm{R}$ onto the interval $\mathcal{I} \in \mathrm{R}$.

Following Ref. $\mathrm{B}$, one can consider symmetric maps of the form shown in Fig. 1,

$$
f(x)=\tanh (A x)-B \tanh (C x)
$$

with $A=20, B=1.5$ and $C=2$ and where $\mathcal{S} \equiv R$ and $\mathcal{I}=[-a, a]$ ( $a \simeq 0.684$ ). The presence of the noise term of amplitude $\sigma$ will obviously enlarge the support of the invariant measure to the interval $[-a-\sigma, a+\sigma]$. This will be not a problem for map (2) since $\mathcal{S}$ is thw whole real axis. So far, the corresponding CML model is certainly not one of the most investigated

papero: submitted to World Scientific on July 6, 2018 


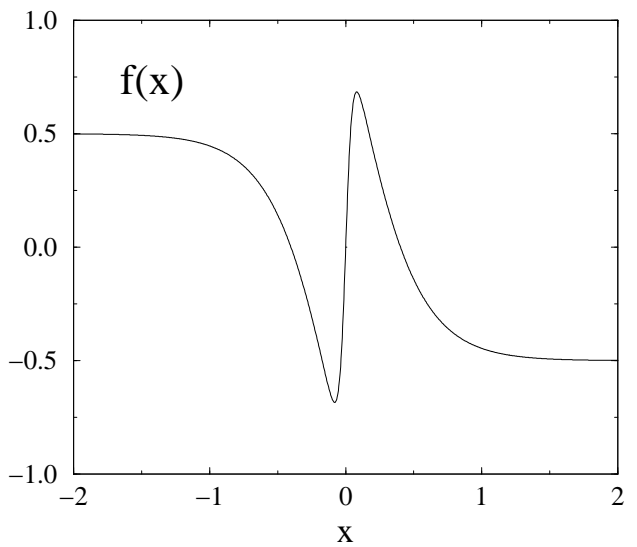

Figure 1. The map $f(x)=\tanh (A x)-B \tanh (C x)$ for $A=20, B=1.5$ and $C=2$.

problems in the literature. In fact, CML are usually defined using maps $f$ of the interval $[0,1]$ onto itself, like the logistic map, that are known to exhibit specific dynamical features, yielding many interesting statistical and dynamical effects in the presence of diffusive coupling (e.g., see Ref. 3.12,13). In this case $\mathcal{I} \equiv \mathcal{S}=[0,1]$ and the dynamic rule (11) can maintain the state variable $x_{i}^{t}$ inside $\mathcal{S}$ only by adopting some further recipe: for instance $x_{i}^{t+1} \rightarrow$ $x_{i}^{t+1}+1\left(x_{i}^{t+1} \rightarrow x_{i}^{t+1}-1\right)$ if $x_{i}^{t+1}<0\left(x_{i}^{t+1}>1\right)$. This rule modifies the probability measure of the state variable $x_{i}^{t}$ w.r.t. the noise-free case. Herzel and Freund 5 showed that, in low-dimensional systems, a similar recipee breaks the symmetry of the noise process, giving rise to an effective statedependent noise, with nonvanishing mean. Later, Lai and Zhou 6 have shown that, even maintaining symmetric noise, synchronization can be achieved, provided sufficiently large noise amplitudes amplify the role of contracting regions in phase space.

This notwithstanding, for what concerns synchronization in high dimensional dynamical systems like CML, it is more interesting to point out that the above mentioned recipee introduces effective discontinuities. In fact, two state variables, whose values are close to one another and to one of the boundaries, can be driven by the addition of spatio-temporal noise at a distance of order 1. This amounts to a strong non-linear effect, that is highly reminiscent of the mechanism yielding stable chaos in CML (see Ref. 19 ). Here it is induced by noise, while in the just quoted CML model the discontinuity was built in 
the map $f$. As we are going to show in Sec. 3, the only distinctive feature of these scenarios is that the final attractor is a stable periodic orbit in the deterministic CML and a stochastic one, in the noisy model.

In the following section we discuss synchronization in both of the above mentioned models of noise-driven CML. For this purpose, here it is worth defining the main quantitative indicators that we have used for working out our analysis. A first sensible quantity is the maximum Lyapunov exponent, $\Lambda$, measured according to the method outlined in Ref. 6 . We want to remark that this quantity is defined for deterministic dynamics (i.e. the noise-free case, $\sigma=0$ ) and it measures the average exponential expansion rate of nearby trajectories within a linear stability analysis. For $\sigma \neq 0$ we are faced with stochastic trajectories and it is not a priori obvious that $\Lambda$ is still a well-defined quantity. On the other hand, the linearized evolution equations in the tangent space of Eq. (11) do not depend explicitely on $\eta_{i}^{t}$. Accordingly, they are formally equivalent for both the noisy and the noise-free cases. However, the presence of noise modifies the dynamical evolution of the system and therefore also the tangent space dynamics. Careful numerical simulations show that $\Lambda$ remains a well defined asymptotic quantity, measuring the exponential expansion rate of infinitesimal perturbations of the stochastic evolution. In particular, its value is found to depend on $\sigma$, but not on the realization of noise.

Another relevant indicator for the study of spatially extended dynamical systems is the average propagation velocity of finite amplitude perturbations 11

$$
V_{F}=\lim _{t \rightarrow \infty} \lim _{L \rightarrow \infty} \frac{\langle F(t)\rangle}{t}
$$

where

$$
F(t)=\max \left\{i: 1 \leq i \leq L ;\left|x_{i}^{t}-y_{i}^{t}\right|>0\right\} .
$$

The trajectories $x_{i}^{t}$ and $y_{i}^{t}$ represent the synchronous evolution according to Eq. (11), with the same realization of noise $\eta_{i}^{t}$, of two initial conditions that differ at time $t=0$ for finite amounts $\delta_{i} \sim \mathcal{O}(1)$ only inside a space region of size $S: y_{i}^{0}=\left[x_{i}^{0}+\delta_{i}\right.$, mod1 $]$ for $|L / 2-i| \leq S$, otherwise $y_{i}^{0}=x_{i}^{0}$.

In the noisy model the average in (3) has to be performed over different initial conditions and noise realizations. It is worth stressing that $V_{F}$ is associated to the nonlinear mechanisms of informatign propagation and it can have a finite value even for non chaotic evolution 10. Accordingly, in order to have no information flow in a spatially extended system not only $\Lambda$ but also $V_{F}$ should vanish.

papero: submitted to World Scientific on July 6, 2018 


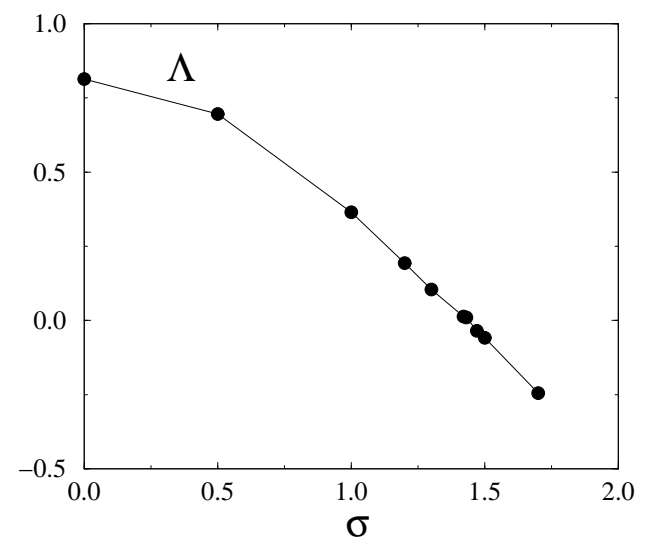

Figure 2. Lyapunov exponent $\Lambda$ as a function of the amplitude of the noise $\sigma$ for a chain of 1000 coupled maps of the type reported in Fig. 1 for $\varepsilon=0.1$. The $\Lambda$-values have been obtained by integrating for 130,000 time steps.

\section{Synchronization of Replicae}

Two natural quantities can be introduced for identifying the synchronization of replicae: the first passage time $\tau_{1}(\Delta)$, i.e. the time needed for two orbits, starting from different initial conditions $x_{i}^{0}$ and $y_{i}^{0}$, to approach each other at a distance $d(t)=\frac{1}{N} \sum_{i=1}^{N}\left|x_{i}^{t}-y_{i}^{t}\right|$ smaller than a given threshold $\Delta$ (usually assumed much smaller than unity); the time $\tau_{2}(\Delta)$ during which their distance remains smaller than $\Delta 14$. Since, in general, these times should depend on the initial conditions and on the noise realizations, in what follows we shall use the same symbols for denoting the averaged quantities. In all the numerical calculations hereafter reported we have used $\Delta \sim 10^{-8}-10^{-10}$. We have also verified that results are not affected by the choice of $\Delta$, provided it is small enough, tipically $\Delta<10^{-7}$.

As a first example let us consider the CML with the map $f$ shown in Fig. 1. In this case, we have observed synchronization for all the $\varepsilon$-values that we tested.

For instance, with a diffusive coupling value $\varepsilon=0.1$ we have found that synchronization is obtained for $\sigma>\sigma_{s}=1.44$. In Fig. 2 we show that $\Lambda$ is a function of $\sigma$ and it becomes negative at $\sigma_{s}$. This is the first evidence of the validity of Pikovsky's criterion also for spatially extended systems.

In principle, one has to consider that $\tau_{1}(\Delta)$ is also a function of the system

papero: submitted to World Scientific on July 6, 2018 
size $L$. For $\sigma<\sigma_{s}$, i.e. $\Lambda>0$, we find that $\tau_{1}(\Delta)$ diverges exponentially with the system size $L: \tau_{1}(\Delta) \sim e^{L / \xi}$. The scale factor $\xi(\varepsilon, \sigma)$ plays the role of an effective correlation length and is found to be finite for any choice of the parameters 15 . This exponential divergence can be explained by a simple argument. Despite the spatial coupling, the combined effect of chaos and noise makes an effective number of degrees of freedom, $L / \xi$, in the replicae evolve independently. Accordingly, the probability that the replicae get closer than a distance $\Delta$ should be proportional to $\Delta^{L / \xi}$ and $\tau_{1}(\Delta)$ can be reasonably assumed to be inversely proportional to this probability.

At variance, a logarithmic dependence of $\tau_{1}(\Delta)$ on $L$ is found for $\sigma>\sigma_{s}$. Numerical simulations show that the number of regions made of a few synchronized sites increases as time flows. Moreover, once a synchronized region is formed, it grows linearly in time, until all regions merge and synchronization sets in over the whole lattice. Upon these observations, one can introduce a simple model accounting for the logarithmic dependence of $\tau_{1}$ on $L$. An effective rate equation for the number of synchronized sites, $n(t)$, can be constructed by assigning a probability $p$ for the formation of new synchronized sites and a rate $\gamma$ for the linear increase of synchronized regions:

$$
\frac{\partial n}{\partial t}=\gamma+p(L-n)
$$

with $0 \leq n(t) \leq L$. Solving this equation with the initial condition $n(0)=0$, one obtains an estimate of $\tau_{1}$ by imposing the condition $n\left(\tau_{1}\right)=L$ :

$$
\tau_{1}=\frac{1}{p} \ln \left[\frac{p L}{\gamma}+1\right] .
$$

Note that a logarithmic dependence with $L$ (see, e.g., the inset of Fig. (1) is consistent with the condition $\frac{p L}{\gamma}>>1$, that can be always achieved for sufficiently large values of $L$.

For what $V_{F}$ is concerned, in this case it is not expected to provide any additional information w.r.t. $\Lambda$. Actually, $V_{F}$ is found to be positive for $\sigma<\sigma_{s}$, while it vanishes for $\sigma>\sigma_{s}$.

Different scenarios are obtained by considering the second kind of noisy CML described in the previous section. Here we consider the logistic map at the Ulam point

$$
f(x)=4 x(1-x)
$$

Let us first study the case $\varepsilon=1 / 3$ : for large enough values of $L, \Lambda$ and $V_{F}$ are positive for any $\sigma$. However, their values are found to vary with $\sigma$, as well $\tau_{1}$ and $\tau_{2}$ that remain finite up to $\sigma \sim \mathcal{O}(1)$. Again, $\tau_{1}$ is found to diverge exponentially with $L$.

papero: submitted to World Scientific on July 6, 2018 


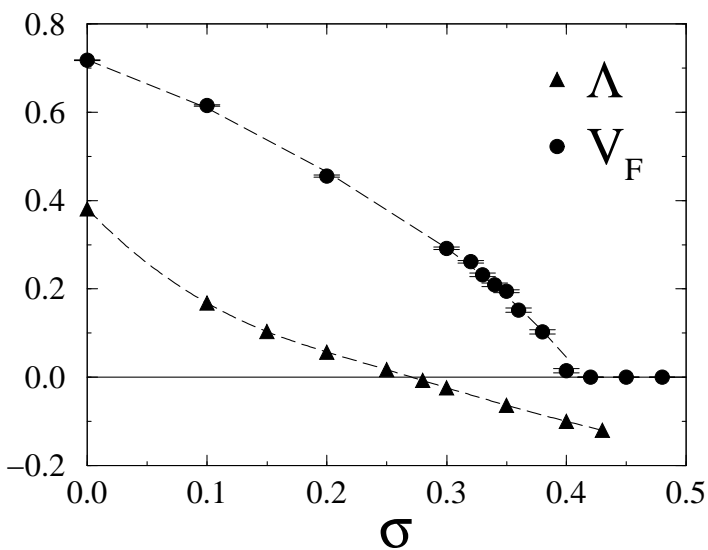

Figure 3. Behaviour of the propagation velocity $V_{F}$ (circles) and of the maximum Lyapunov exponent $\Lambda$ (triangles) versus noise amplitude $\sigma$ for coupled logistic maps with $\varepsilon=2 / 3$. Both quantities have been computed for $L=1024$, averaging over $10^{3}$ initial conditions each one followed for $\mathcal{O}\left(10^{5}\right)$ time steps. The dashed lines are a guide for the eyes.

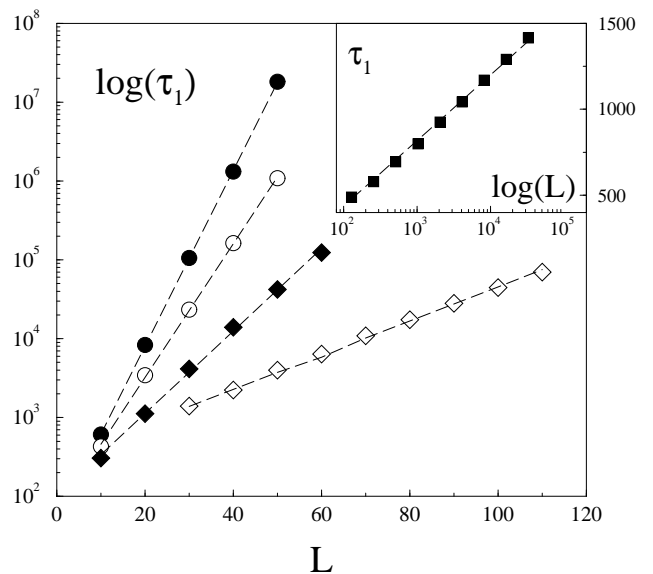

Figure 4. Exponential scaling of $\tau_{1}$ versus $L$ for coupled logistic maps with $\varepsilon=2 / 3$ at different values of the noise amplitude: $\sigma=0.3$ (empty circles), 0.32 (filled circles), 0.35 (empty diamonds), 0.38 (filled diamonds). The inset shows the logarithmic scaling of $\tau_{1}$ versus $L$ for $\sigma=0.45$. The values of $\tau_{1}$ have been computed with $\Delta=10^{-10}$ and averaged over $10^{3}$ initial conditions. 


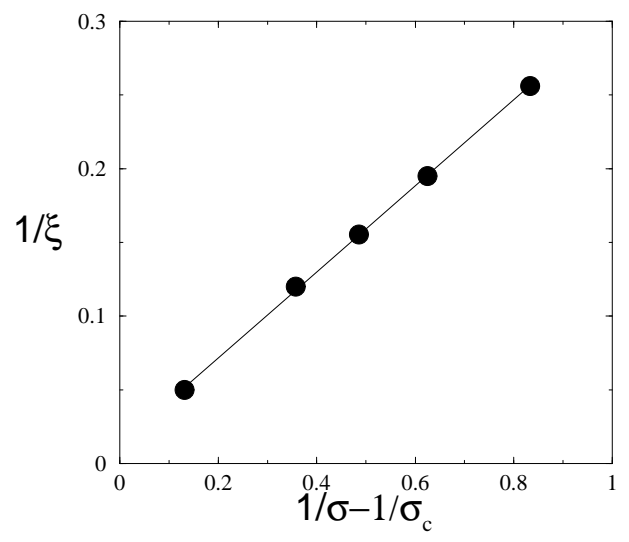

Figure 5. Inverse of the scale factor $1 / \xi$ as a function of $\sigma^{-1}-\sigma_{c}^{-1}\left(\sigma_{c}=0.4\right)$ for CML logistic maps with $\varepsilon=2 / 3$ and $0.30 \leq \sigma \leq 0.38$.

A new interesting scenario occurs for $\varepsilon=2 / 3$, where, for $L \geq \mathcal{O}(10), \Lambda$ is negative for $\sigma>0.27$ (see Fig. 3). Therefore, one expects that synchronization occurs and this is indeed the case although, for $0.27<\sigma<0.4, \tau_{1}(\Delta)$ is still found to increase exponentially with $L$ (see Fig. 4). On the other hand, for $\sigma>0.4 \tau_{1}(\Delta)$ grows logarithmically with $L$ (see the inset of Fig. 4).

This transition between two completely different dynamical regimes is ruled by $V_{F}$, that drops to zero at $\sigma_{c} \approx 0.4$ (see Fig. 3). In fact, for $\sigma<\sigma_{c}$, a positive $V_{F}$ implies that any perturbation of finite amplitude propagates through the lattice. Despite the linear mechanism is no more active $(\Lambda<0)$, we find that the non-linear mechanism of information propagation maintains the exponential dependence of $\tau_{1}$ on $L$, thus still implying that an effective number of degrees of freedom evolves independently. In particular, we find numerical evidence of the relation $\xi^{-1} \propto\left(\sigma^{-1}-\sigma_{c}^{-1}\right)$ for $\sigma<\sigma_{c}$ (see Fig. 同); this indicates that the scale factor $\xi$ diverges at the transition point.

Eventually, only when $d(t)$ becomes sufficiently small the linear mechanism acts as a stabilizing factor on the dynamics of the replicae and synchronization is achieved.

For $\sigma>0.4$, where also the nonlinear mechanism is absent, numerical simulations exhibit the same scenario described in the "symmetric map" CML for $\sigma>\sigma_{s}$.

One can counclude that in this last case the conditions $\Lambda<0$ and $V_{F}=0$ have to be fulfilled independently for guaranteeing noise-driven synchroniza- 
tion of two replicae within a "reasonable" time span in a CML of large but finite size $L$.

We want to point out that this scenario is not peculiar of the logistic map for $\varepsilon=2 / 3$. Actually, we have verified that it holds in a finite interval of values around $\varepsilon=2 / 3$. Moreover, a very similar situation is pbtained when considering dynamics (1) for a model of period-3 stable maps 10

$$
f(x)= \begin{cases}b x & 0<x<1 / b \\ a+c(x-1 / b) & 1 / b<x<1\end{cases}
$$

where $b=2.7, a=0.07$ and $c=0.1$. This case is particularly interesting for a twofold reason. It has been shown that the noise-free case has a negative $\Lambda$ for any value of $\varepsilon$ and, moreover, it exhibits a peculiar transition at $\varepsilon_{c} \approx$ 0.6 from a frozen disordered phase with $V_{F}=0$ to a chaotic phase with $V_{F}>0$ 16. At variance with the case of coupled logistic maps, when noise is added $\Lambda$ remains negative for any value of $\sigma$. The phase transition disappears, because, independently of $\varepsilon, V_{F}$ is found to be positive for very small noise amplitudes. On the other hand, by increasing $\sigma$ up to a critical value $\sigma_{c}(\varepsilon)$, $V_{F}$ is found to drop again to zero not only below, but also above $\varepsilon_{c}$ : for instance, one has $\sigma_{c} \approx 0.16$ for $\varepsilon=0.58$ and $\sigma_{c} \approx 0.18$ for $\varepsilon=0.62$. In both of these cases we have recovered the same kind of mechanisms characterizing the synchronization transition discussed for the case of coupled logistic maps with $\varepsilon=2 / 3$ (see Fig. 6). In this sense, it seems reasonable to conjecture that this phenomenon is present in a wide class of spatially extended dynamical systems and that it can be characterized making use of the same kind of analysis discussed in this paper for CML models.

As a final remark, we would like to stress that all this scenario is reminiscent of the phenomenology associated to stable chaos in CML, that was identified for model (8) 10 . Also in that case unpredictability was characterized by transients increasing exponentially with $L$ when $V_{F}>0$. The analogy is strenghtened by the fact that additive noise induces "jumps" in the deterministic dynamics of the system, similar to the discontinuities present in model (8). As a matter of fact, the presence of strong variations in the slope of the local map is fundamental in order to observe non-linear propagation mechanisms 17 .

\section{Acknowledgments}

We want to thank F. Bagnoli and A. Politi for helpful discussions. Part of this work was performed at the Institute of Scientific Interchange in Torino, during the workshop on "Complexity and Chaos", June 1998. We acknowl-

papero: submitted to World Scientific on July 6, 2018 


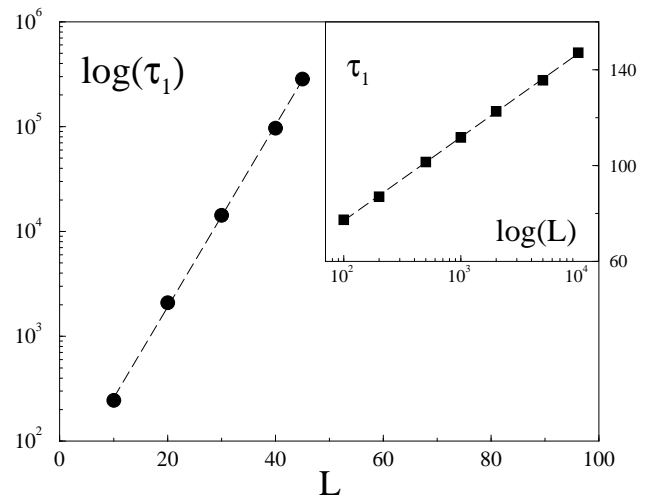

Figure 6. Exponential scaling of $\tau_{1}$ versus $L$ for coupled stable maps with $\varepsilon=0.31$ and $\sigma$ $=0.15$ (filled circles). The inset shows the logarithmic scaling of $\tau_{1}$ versus $L$ for $\sigma=0.25$ (filled squares). The values of $\tau_{1}$ have been computed with $\Delta=10^{-8}$ and averaged over $10^{3}$ initial conditions.

edge CINECA in Bologna and INFM for providing us access to the parallel computer CRAY T3E under the grant "Iniziativa Calcolo Parallelo".

\section{References}

1. T. Halpin-Haley and Y.-C. Zhang, Phys. Rep. 254, 215 (1995); A.-L. Barabasi and H.E. Stanley Fractal Concepts in Surface Growth (Cambridge University Press, Cambridge, 1995).

2. M. Kardar, G. Parisi and Y.-C. Zhang, Phys. Rev. Lett. 56, 889 (1986); S. Zalesky, Physica D, 34, 427 (1989); K. Sneppen, J.Krug, M.H. Jensen, C. Jayaprakash, and T. Bohr, Phys. Rev. A 46, R7351 (1992).

3. R. Kapral, R. Livi and A. Politi, Phys. Rev. Lett. 79, 2277 (1997).

4. S. Fahy and D.R. Hamann, Phys. Rev. Lett. 69, 761 (1992); A. Maritan and J. R. Banavar, Phys. Rev. Lett. 72, 1451 (1994) and 73, 2932 (1994).

5. H. Herzel and J. Freund, Phys. Rev. E 52, 3238 (1995).

6. C.H. Lai and Changsong Zhou, Europhys. Lett. 43, 376 (1998).

7. A. S. Pikovsky, Phys. Lett. A 165, 33 (1992); Phys. Rev. Lett. 73, 2931 (1994).

8. K. Kaneko, Prog. Theor. Phys. 72, 980 (1984); I. Waller and R. Kapral, Phys. Rev. A 30,2047 (1984). 
9. I. Shimada and T. Nagashima, Prog. Theor. Phys. 61, 1605 (1979); G. Benettin, L. Galgani, A. Giorgilli and J.M. Strelcyn, Meccanica, March 15 and 21 (1980).

10. A. Politi, R. Livi, R. Oppo, and R. Kapral, Europhys. Lett. 22, 571 (1993); R. Kapral, R. Livi, R. Oppo, and A. Politi, Phys. Rev. E 49, 2009 (1194).

11. P. Grassberger, Physica Scripta, 40, 1033 (1985); K. Kaneko, Physica D, 23, 436 (1986).

12. K. Kaneko, Prog. Theor. Phys. 74, 1033 (1985).

13. H. Chaté and P. Manneville, Phys. Rev. Lett. 58, 112 (1987).

14. We have checked that different norms, e.g. the maximum or the euclidean ones, do not modify the resulting scenario. Note that both quantities are well defined also in the noise-free case, since there is a finite probability that two different initial conditions may stay closer than a finite distance $\Delta$.

15. Although intricate, the dependence of $\xi$ on the parameters does not add any relevant feature to the outlined scenario. For the sake of space it will be discussed in a forthcoming paper, containing more details and more examples than those reported in this letter.

16. F. Cecconi, R. Livi and A. Politi, Phys. Rev. E 57, 2703 (1998).

17. A. Torcini, P. Grassberger and A. Politi, J. Phys. A : Math. Gen. 27, 4533 (1995). 